\title{
Effects of systematic rehabilitation programs on quality of life in patients undergoing lung resection
}

\author{
XU-HONG LI ${ }^{1}$, JIA-LIANG ZHU ${ }^{2}$, CAO HONG $^{2}$, LEI ZENG ${ }^{2}$, LI-MING DENG ${ }^{1}$ and LONG-YU JIN ${ }^{2}$ \\ Departments of ${ }^{1}$ Rehabilitation and ${ }^{2}$ Cardiothoracic Surgery, The Third Xiangya Hospital \\ of Central South University, Changsha, Hunan 410013, P.R. China
}

Received August 6, 2012; Accepted September 24, 2012

DOI: $10.3892 /$ mco.2012.31

\begin{abstract}
The aim of this study was to investigate the effects of systematic rehabilitation programs on the quality of life (QOL) in patients undergoing lung resection of malignant lung lesions. In this prospective population-based cohort study, QOL in patients prior to, as well as 3 and 6 months after surgery, was investigated. Using a single-group design, 48 patients ( 7 females and 41 males) with suspected operable lung cancer were included in this study. The demographic characteristics and the clinical history of the patients were recorded. QOL [assessed using the European Organization for Research and Treatment of Cancer Quality of Life Questionnaire Core 3.0 (EORTC QLQ-C30)] was evaluated at baseline (immediately before), and 3 and 6 months after surgical resection. The systematic rehabilitation program, including breathing control, breathing exercises, relaxation training, upper and lower extremity exercises, mobilization and additional incorporating physiotherapy programs, was designed to meet each patient's individual needs. The $\chi^{2}$ and Fisher's tests showed no statistically significant difference in the two groups in terms of age, gender, behavior, clinical stage, adjuvant therapy and Karnofsky scores. QOL analysis of baseline was homogeneous between the experimental and control groups. Three months after the rehabilitation process, the experimental group demonstrated an increase in the general QOL functional scales and a decrease of symptom scales compared to the control group. These changes were statistically significant in the functional scales of global health $(\mathrm{P}<0.01)$, physical function $(\mathrm{P}<0.01)$, role function $(\mathrm{P}<0.01)$, emotional function $(\mathrm{P}<0.05)$, symptom scales of fatigue $(\mathrm{P}<0.01)$ and appetite loss $(\mathrm{P}=0.001)$. Six months after the intervention, the outcome was the same as 3 months after the intervention in
\end{abstract}

Correspondence to: Professor Long-Yu Jin, Department of Cardiothoracic Surgery, The Third Xiangya Hospital of Central South University, Tongzipo Road 138, Changsha, Hunan 410013, P.R. China

E-mail: jinlongyu1123@163.com

Key words: systematic rehabilitation programs, quality of life, lung cancer, European Organization for Research and Treatment of Cancer Quality of Life Questionnaire version 3.0 functional scale domains. However, in the symptom scales, the symptoms in the experimental group were improved compared to the control group. The domains had been significant in the scales of fatigue $(\mathrm{P}<0.001)$, dyspnea $(\mathrm{P}<0.001)$, pain $(\mathrm{P}<0.001)$, insomnia $(\mathrm{P}<0.001)$, appetite loss $(\mathrm{P}<0.001)$ and constipation $(\mathrm{P}<0.001)$. Therefore, the two groups demonstrated a statistically significant difference in 10 domains. In addition, the experimental group demonstrated a significant recovery. In conclusion, systematic rehabilitation programs may be beneficial for lung cancer patients by reducing respiratory symptoms, pain, and improving health-related QOL. Consequently, the findings of this study suggest that systematic rehabilitation programs, prepared by taking into consideration the individual requirements of lung cancer patients, should be incorporated into lung cancer treatment.

\section{Introduction}

Lung cancer constitutes the leading cause of cancer mortality and morbidity worldwide (1). The incidence of lung cancer has markedly increased over the last decades in China. Surgery is considered to be the treatment that offers the greatest potential for cure for these patients (2). Pulmonary resection has a direct negative impact on pulmonary function and quality of life (QOL), especially on the QOL related to aspects directly linked to pulmonary function. The aim of the present study was to evaluate the effects of a systematic rehabilitation program on the QOL in patients undergoing lung resection of malignant lung lesions.

\section{Patients and methods}

Patients. Between April 2008 and May 2011, 56 consecutive lung cancer patients with non-small cell lung cancer (NSCLC) were enrolled in this study. Of the 56 cases, 48 underwent different modes of surgery including pneumonectomy, lobectomy/bilobectomy or sleeve lobectomy via open thoracotomy and 8 cases were excluded from this study for non-compliance with surgical treatment or were rejected. Patients $(n=48)$ comprised 41 males and 7 females, with a mean age of 56 years (range, 41-75 years). The present study was approved by the Research Ethics Committees of the Third Xiangya Hospital of the Central South University (Changsha, China). Written informed consent was provided by the patients included. 
Table I. Patient characteristics.

\begin{tabular}{|c|c|c|c|c|}
\hline \multirow[b]{2}{*}{ Characteristics } & \multicolumn{2}{|c|}{ Groups } & \multirow[b]{2}{*}{ Statistical value } & \multirow[b]{2}{*}{ P-value } \\
\hline & Experimental & Control & & \\
\hline \multicolumn{5}{|l|}{ Age (years) } \\
\hline Range & $41-75$ & $42-74$ & & \\
\hline Mean \pm SD & $57.2 \pm 8.9$ & $55.9 \pm 8.5$ & $\mathrm{t}=0.517$ & 0.304 \\
\hline \multicolumn{5}{|l|}{ Gender } \\
\hline Male & 20 & 19 & & \\
\hline Female & 4 & 5 & $\chi^{2}=0.137$ & 0.712 \\
\hline \multicolumn{5}{|l|}{ Behavior } \\
\hline Smoking & 16 & 18 & & \\
\hline No-smoking & 8 & 6 & $\chi^{2}=0.403$ & 0.525 \\
\hline \multicolumn{5}{|l|}{ Clinical stage } \\
\hline II & 7 & 8 & & \\
\hline IIIa & 12 & 11 & & \\
\hline IIIb & 5 & 5 & $\chi^{2}=0.110$ & 0.946 \\
\hline \multicolumn{5}{|l|}{ Adjuvant therapy } \\
\hline Concurrent chemotherapy & 15 & 16 & & \\
\hline Concurrent chemoradiotherapy & 9 & 8 & $\chi^{2}=0.091$ & 0.763 \\
\hline \multicolumn{5}{|l|}{ Karnofsky score } \\
\hline Range & $60-90$ & $60-90$ & & \\
\hline Median & 80 & 80 & $\mathrm{Z}=0.010^{\mathrm{a}}$ & 0.992 \\
\hline
\end{tabular}

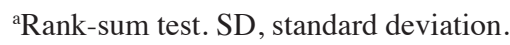

Methods. The participants received standard medical and nursing care involving a clinical pathway. Certain patients received 4-6 cycles of standard chemotherapy. Radiation therapy was delivered by a 6 megavolt $(\mathrm{MeV})$ photon linear accelerator following a standard protocol. Radiation was administered by $2.0 \mathrm{~Gy}$ daily fractions, 5 times/week at a total dose of $60 \mathrm{~Gy} / 6$ weeks. The experimental group received the systematic rehabilitation program, including breathing control, breathing exercises, relaxation training, progressive exercises and additional incorporating physiotherapy programs during the preoperative period until discharge and at 6 months after the surgical procedure. The patients completed health-related QOL parameters prior to as well as 3 and 6 months after surgery. QOL was assessed using the Chinese version of the European Organization for the Research and Treatment of Cancer Quality of Life Questionnaire Core 30 (EORTC QLQ-C30) (3), which investigates a patient's ability to fulfill the activities of everyday life. EORTC QLQ-C30 is a 30-entry cancer-specific questionnaire that incorporates six functioning scales (Global, physical, role, cognitive, emotional and social), nine symptom scales (fatigue, pain, nausea/vomiting, dyspnea, insomnia, loss of appetite, constipation, diarrhea and financial difficulties), and one global health status/QOL scale. The measures are scored from 0 to 100. For the functional and global health status/QOL scales, a high value reflects a better level of functioning, while a low value is representative of low or disappointing function. However, higher scores of the symptom scales indicate more severe problems.
Statistical analysis. Data were analyzed using the biological statistics analysis software SPSS 13.0 Package. Application of the constituent ratio of the $\chi^{2}$ test/Fisher's test was used for data analysis. QOL score prior to treatment was compared using the Student's t-test, while longitudinal data were compared using repeated measures analysis of variance (ANOVA). $\mathrm{P}<0.05$ was considered to indicate a statistically significant difference.

\section{Results}

Patient characteristics. A total of 48 patients, classified into experimental and control groups, were included in this study. The average age of the patients in the experimental group was 57 years and in the control group 55 years. The $\chi^{2}$ and Fisher's tests showed no statistically significant difference between the two groups in terms of age, gender, behavior, clinical stage, adjuvant therapy and Karnofsky scores. The two groups were well-balanced concerning patient characteristics (Table I). The patients underwent surgery, 21 patients received concurrent chemotherapy, 16 external-beam radiation therapy and 11 concurrent chemoradiotherapy.

Analysis of QOL at baseline. Basic QOL on the scale of 6 functional areas and 4 symptom domains were compared prior to treatment in the two groups. Analysis of the results prior to intervention showed that the two groups were homogeneous concerning QOL (Table II). 
Table II. QOL baseline scores of the two patient groups (mean $\pm \mathrm{SD})$.

\begin{tabular}{lccc}
\hline & \multicolumn{2}{c}{ Groups } & \\
\cline { 2 - 4 } EORTC QLQ-C30 scales & Experimental & Control & t-value \\
\hline Function & & & -0.288 \\
Global & $58.3 \pm 26.3$ & $60.3 \pm 21.6$ & -0.140 \\
Physical & $64.3 \pm 22.4$ & $65.4 \pm 31.2$ & -0.057 \\
Role & $60.2 \pm 30.9$ & $60.7 \pm 30.4$ & -0.083 \\
Emotional & $45.6 \pm 36.6$ & $46.5 \pm 38.2$ & 0.434 \\
Cognitive & $64.0 \pm 21.4$ & $60.7 \pm 30.5$ & 0.579 \\
Social & $70.2 \pm 25.0$ & $66.4 \pm 20.2$ & 0.478 \\
Symptom & & & 0.467 \\
Fatigue & $20.8 \pm 13.5$ & $26.3 \pm 9.8$ & 0.333 \\
Nausea/vomiting & $35.6 \pm 14.1$ & $42.7 \pm 12.6$ & -1.615 \\
Pain & $42.3 \pm 21.2$ & $38.5 \pm 17.6$ & -0.863 \\
Dyspnea & $18.2 \pm 19.7$ & $16.9 \pm 18.7$ & 0.676 \\
Insomnia & $9.4 \pm 8.7$ & $11.2 \pm 10.4$ & 0.283 \\
Appetite loss & $27.0 \pm 5.4$ & $24.5 \pm 7.3$ & -0.650 \\
Constipation & $10.4 \pm 6.5$ & $12.0 \pm 7.8$ & 1.349 \\
Diarrhea & $12.3 \pm 9.8$ & $10.8 \pm 9.2$ & 0.057 \\
Financial difficulties & $10.6 \pm 8.2$ & $9.5 \pm 6.8$ & 0.196 \\
\hline
\end{tabular}

QOL, quality of life; SD, standard deviation; EORTC QLQ-C30, European Organization for Research and Treatment of Cancer Quality of Life Questionnaire Core 3.0.

Table III. Functional and symptom outcomes derived from EORTC QLQ-C30 in the experimental and control groups 3 months after the intervention (mean $\pm \mathrm{SD})$.

\begin{tabular}{lcccr}
\hline & \multicolumn{2}{c}{ Groups } & & \\
\cline { 2 - 3 } EORTC QLQ-C30 scales & Experimental & Control & & P-value \\
\hline Function & & & & \\
Global & $78.6 \pm 24.7$ & $58.1 \pm 22.3$ & 3.018 & 0.002 \\
Physical & $83.8 \pm 24.9$ & $64.2 \pm 21.5$ & 2.913 & 0.003 \\
Role & $76.6 \pm 20.4$ & $60.2 \pm 19.8$ & 2.826 & 0.003 \\
Emotional & $64.5 \pm 21.8$ & $50.6 \pm 18.6$ & -0.376 & 0.452 \\
Cognitive & $75.6 \pm 34.3$ & $76.7 \pm 28.0$ & 0.713 & 0.240 \\
Social & $70.0 \pm 22.3$ & $65.6 \pm 20.4$ & & 0.006 \\
Symptom & & & -2.643 & 0.322 \\
Fatigue & $18.3 \pm 10.5$ & $30.2 \pm 19.4$ & 0.466 & 0.294 \\
Nausea/vomiting & $40.2 \pm 22.8$ & $36.7 \pm 28.9$ & -0.544 & 0.127 \\
Pain & $30.8 \pm 25.5$ & $34.3 \pm 18.5$ & -1.153 & 0.468 \\
Dyspnea & $35.6 \pm 14.1$ & $41.4 \pm 20.2$ & 0.0080 & 0.001 \\
Insomnia & $37.2 \pm 15.0$ & $36.9 \pm 10.8$ & -3.139 & 0.334 \\
Appetite loss & $4.2 \pm 4.7$ & $12.1 \pm 11.4$ & 0.432 & 0.083 \\
Constipation & $8.4 \pm 4.6$ & $7.5 \pm 9.1$ & -1.408 & 0.251 \\
Diarrhea & $22.0 \pm 8.5$ & $25.6 \pm 9.2$ & -0.676 & \\
Financial difficulties & $12.4 \pm 10.9$ & $14.7 \pm 12.6$ & & \\
\hline
\end{tabular}

EORTC QLQ-C30, European Organization for Research and Treatment of Cancer Quality of Life Questionnaire Core 3.0; SD, standard deviation.

Analysis of QOL at 3 months after the rehabilitation process. Three months after the rehabilitation process, the experimental group demonstrated an increase in the general
QOL functional scales and a decrease of symptom scales compared to the control group. These changes were statistically significant in the functional scales of global health 
Table IV. Functional and symptom outcomes derived from EORTC QLQ-C30 in the experimental and control groups 6 months after the intervention (mean $\pm \mathrm{SD}$ ).

\begin{tabular}{lcccr}
\hline & \multicolumn{2}{c}{ Groups } & & \\
\cline { 2 - 3 } EORTC QLQ-C30 scales & Experimental & Control & t-value & P-value \\
\hline Function & & & & 0.001 \\
Global & $80.2 \pm 22.5$ & $56.4 \pm 25.3$ & 3.444 & 0.005 \\
Physical & $84.5 \pm 28.7$ & $64.5 \pm 22.6$ & 2.682 & 0.001 \\
Role & $86.2 \pm 24.5$ & $63.4 \pm 21.0$ & 3.461 & 0.007 \\
Emotional & $68.4 \pm 21.2$ & $54.4 \pm 16.5$ & 2.553 & 0.334 \\
Cognitive & $78.5 \pm 22.4$ & $75.4 \pm 27.2$ & 0.431 & 0.343 \\
Social & $86.7 \pm 43.3$ & $82.0 \pm 36.3$ & 0.408 & 0.000 \\
Symptom & & & & 0.071 \\
Fatigue & $10.2 \pm 7.2$ & $40.3 \pm 16.5$ & -8.191 & 0.000 \\
Nausea/vomiting & $20.5 \pm 14.1$ & $26.4 \pm 13.2$ & -1.496 & 0.000 \\
Pain & $18.7 \pm 16.5$ & $38.6 \pm 20.7$ & -3.683 & 0.000 \\
Dyspnea & $16.4 \pm 8.2$ & $46.5 \pm 20.1$ & -6.793 & 0.000 \\
Insomnia & $15.6 \pm 12.8$ & $38.2 \pm 21.6$ & -4.410 & 0.000 \\
Appetite loss & $2.1 \pm 1.2$ & $9.7 \pm 8.2$ & -4.493 & 0.083 \\
Constipation & $2.5 \pm 1.3$ & $9.2 \pm 6.8$ & -4.741 & 0.251 \\
Diarrhea & $22.0 \pm 8.5$ & $25.6 \pm 9.2$ & -1.408 & -0.676 \\
Financial difficulties & $12.4 \pm 10.9$ & $14.7 \pm 12.6$ & & \\
\hline
\end{tabular}

EORTC QLQ-C30, European Organization for Research and Treatment of Cancer Quality of Life Questionnaire Core 3.0; SD, standard deviation.

Table V. EORTC QLQ-C30 scales of different time and interaction variance analysis results of the two groups.

\begin{tabular}{|c|c|c|c|c|c|c|}
\hline EORTC QLQ-C30 scales & $\mathrm{F}_{\text {Groups }}$ & P-value & $\mathrm{F}_{\text {Time }}$ & P-value & $\mathrm{F}_{\text {Group } \mathrm{x} \mathrm{time}}$ & P-value \\
\hline \multicolumn{7}{|l|}{ Function } \\
\hline Global & 9.332 & 0.004 & 10.659 & 0.000 & 3.190 & 0.046 \\
\hline Physical & 8.652 & 0.005 & 11.337 & 0.000 & 3.668 & 0.029 \\
\hline Role & 10.014 & 0.003 & 9.723 & 0.000 & 3.244 & 0.044 \\
\hline Emotional & 7.568 & 0.008 & 8.352 & 0.000 & 3.874 & 0.024 \\
\hline Cognitive & 2.087 & 0.155 & 2.036 & 0.136 & 3.572 & 0.032 \\
\hline Social & 1.875 & 0.178 & 2.613 & 0.079 & 1.841 & 0.164 \\
\hline \multicolumn{7}{|l|}{ Symptom } \\
\hline Fatigue & 3.557 & 0.066 & 1.899 & 0.156 & 5.294 & 0.007 \\
\hline Nausea/vomiting & 11.229 & 0.002 & 8.664 & 0.000 & 1.211 & 0.303 \\
\hline Pain & 12.364 & 0.001 & 10.256 & 0.000 & 4.968 & 0.009 \\
\hline Dyspnea & 10.872 & 0.002 & 7.681 & 0.001 & 3.179 & 0.046 \\
\hline Insomnia & 9.639 & 0.003 & 6.846 & 0.002 & 1.233 & 0.296 \\
\hline Appetite loss & 12.615 & 0.001 & 3.117 & 0.049 & 3.289 & 0.042 \\
\hline Constipation & 3.243 & 0.078 & 2.699 & 0.073 & 1.657 & 0.196 \\
\hline Diarrhea & 2.068 & 0.157 & 2.897 & 0.060 & 3.605 & 0.031 \\
\hline Financial difficulties & 4.142 & 0.076 & 3.212 & 0.072 & 2.258 & 0.264 \\
\hline
\end{tabular}

EORTC QLQ-C30, European Organization for Research and Treatment of Cancer Quality of Life Questionnaire Core 3.0.

$(\mathrm{P}<0.01)$, physical $(\mathrm{P}<0.01)$, role $(\mathrm{P}<0.01)$ and emotional functions $(\mathrm{P}<0.05)$, symptom scales of fatigue $(\mathrm{P}<0.01)$ and appetite loss $(\mathrm{P}=0.001)$ (Table III).
Analysis of QOL at 6 months after the rehabilitation process. Six months after the intervention, the experimental group demonstrated an increase in the general QOL functional 
scales compared to the control group. These changes were statistically significant in the functional scales of global health $(\mathrm{P}=0.001)$, physical $(\mathrm{P}<0.01)$, role $(\mathrm{P}=0.001)$ and emotional functions $(\mathrm{P}<0.01)$. Regarding symptom scales, the symptoms were also reduced compared to the control group. This decrease was significant in the scales of fatigue $(\mathrm{P}<0.001)$, pain $(\mathrm{P}<0.001)$, dyspnea $(\mathrm{P}<0.001)$, insomnia $(\mathrm{P}<0.001)$, appetite loss $(\mathrm{P}<0.001)$ and constipation $(\mathrm{P}<0.001)$. As a result, the two groups demonstrated a statistically significant difference in 10 domains. The experimental group demonstrated a significant recovery (Table IV).

EORTC QLQ-C30 scales of variance analysis results of the two groups. When compared to the control group, the experimental group demonstrated a statistically significant impact on function scales, such as the global, physical, role and emotional scale at various time points $(\mathrm{P}<0.01)$ and between various time points $(\mathrm{P}<0.001)$. No statistically significant difference was observed in the correlation between groups and time $(\mathrm{P}<0.05)$ (Table V). In addition, no statistically significant differences were observed in the cognitive scale in patients in the two groups and different time points $(\mathrm{P}>0.05)$, while there were statistically significant differences in the correlation between the groups and time $(\mathrm{P}<0.05)$. No statistically significant differences were observed in the social scale in patients in the two groups and different time points, and no statistically significant differences were observed in the correlation between groups and time $(\mathrm{P}>0.05)$ (Fig. 1A-F).

When compared to the control group, the experimental group demonstrated a statistically significant impact on symptom scales, such as nausea/vomiting, pain, dyspnea, insomnia and appetite loss at various time points $(\mathrm{P}<0.01)$ and between various time points $(\mathrm{P}<0.01)$. However, there were no statistically significant differences in the correlation between groups and time $(\mathrm{P}>0.05)$. There were no statistically significant differences in the fatigue, constipation and diarrhea scales in patients in the two groups and various time points $(\mathrm{P}>0.05)$. Moreover, there were statistically significant differences in the correlation of the fatigue, pain, dyspnea, appetite loss and diarrhea scales between groups and time $(\mathrm{P}<0.01)$, while no statistically significant differences were observed in the correlation of nausea/vomiting, insomnia and constipation scales $(\mathrm{P}>0.05)$ (Fig. 1G-O).

\section{Discussion}

Lung cancer is one of the most common malignant tumors. Dysfunction of lung cancer is associated with complex interaction of general and local factors, as well as combined modality therapy (4). These associated therapeutic modalities, advances in surgery, radiotherapy and chemotherapy, as well as cancer itself, continue to yield varied long-term outcomes of adverse physical/functional impairments that markedly reduce a patient's ability to tolerate exercise. Poor exercise tolerance predisposes increased susceptibility to additional common age-related dysfunctions, poor QOL (5) and potential premature death. Therefore, provision of the appropriate treatment and care programs for controlling the disease and improving the patient QOL remains a fundamental issue. A multidimensional therapeutic approach is recommended including pulmonary rehabilitation $(6,7)$ and an adequate level of physical activity $(8,9)$ in order to improve exercise tolerance, health-related QOL as well as surgical candidacy and to decrease surgical morbidity. Recent studies have shown that physical activity may have important benefits on lung cancer patients and survivors (10-12), regardless of the disease stage (13) or limited physical activity $(14,15)$. Limited physical activity, also known as 'de-conditioning', may cause the heart and muscles to regress and become less efficient. Regular exercise is ideal for cancer patients or survivors in general, with recent studies indicating improvements in cardiorespiratory fitness, appetite, cancer-related fatigue and depression (16-18). Individualized exercise rehabilitation programs for postoperative lung cancer patients constitute a great method to improve fitness, which in turn improves cardiac function, as well as oxygen-carrying capacity, physical functioning, muscle strength and endurance. Rehabilitation activity has been shown to improve fitness levels and health status, leading to the improvement of health-related QOL $(19,20)$.

Numerous epidemiological studies have demonstrated that regular physical activity reduces the risk of colon cancer and potentially decreases the risk of endometrium, post- and premenopausal breast cancer, prostate, lung as well as pancreas cancer $(21,22)$. A comprehensive rehabilitation program is essential to improve health status and to enhance QOL in cancer patients. Thus, the questions included concerned the preoperative rehabilitation scheme and the lifetime period of maintenance rehabilitation. No statistically significant difference was observed in patient QOL in the control group during 6 months and it can be indicated that patients in this group were stable, although certain fields, such as dyspnea, they worsened. Prior to rehabilitation intervention, no statistically significant difference was observed between the two groups. However, 3 months after the intervention, the experimental group demonstrated a statistically significant improvement in six fields, while 6 months later, improvement was observed in 11 fields compared to the control group. Therefore, it can be concluded that systematic rehabilitation intervention had a positive effect on the experimental group and that patients' QOL in the experimental group improved significantly. The trend of improving results in the experimental group indicates that time is crucial in assessing the effect of rehabilitation intervention on QOL.

A consistent proportion of patients undergoing lung resection exhibited an important postoperative deterioration of their QOL. Thus, patients who received preoperative counseling and rehabilitation training would benefit from physical and emotional supportive programs, while others demonstrated a higher risk of a relevant physical deterioration and worse mental health, such as pain, pulmonary infection, cardiopulmonary dysfunction, delayed wound healing and slower recovery, decreased physical fitness and QOL after lung resection. Concurrent chemoradiotherapy also induced late side-effects, including organ malfunction, chronic fatigue, pain or premature death in lung cancer survivors. Future systematic rehabilitation knowledge is likely to help lung cancer survivors, their healthcare providers and caregivers by providing evidence for establishing clinical recommendations to enhance their long-term survival and health-related QOL. Thus, the 10 patients in the control group exhibited medical 
A

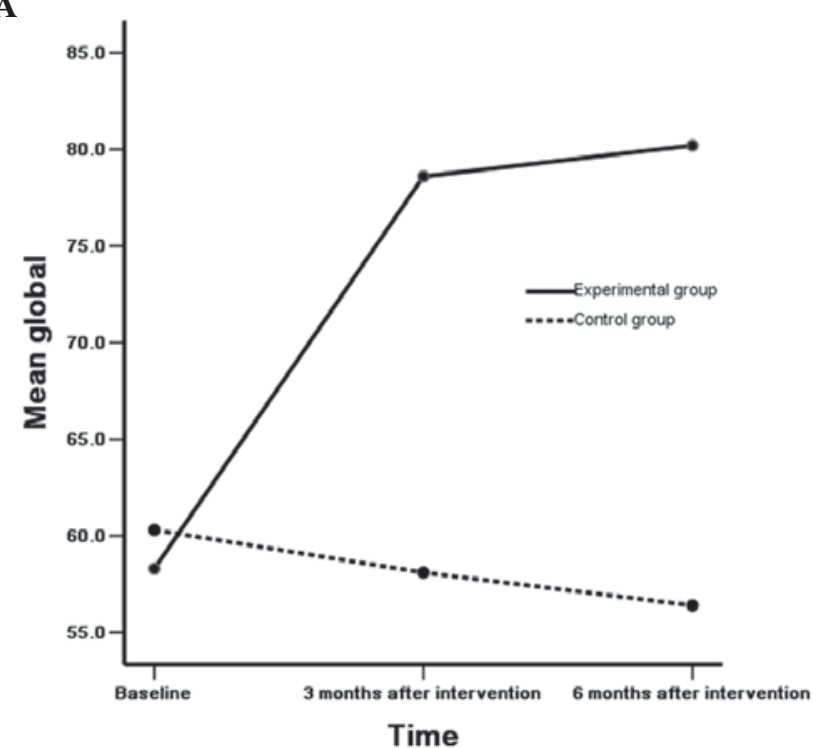

C

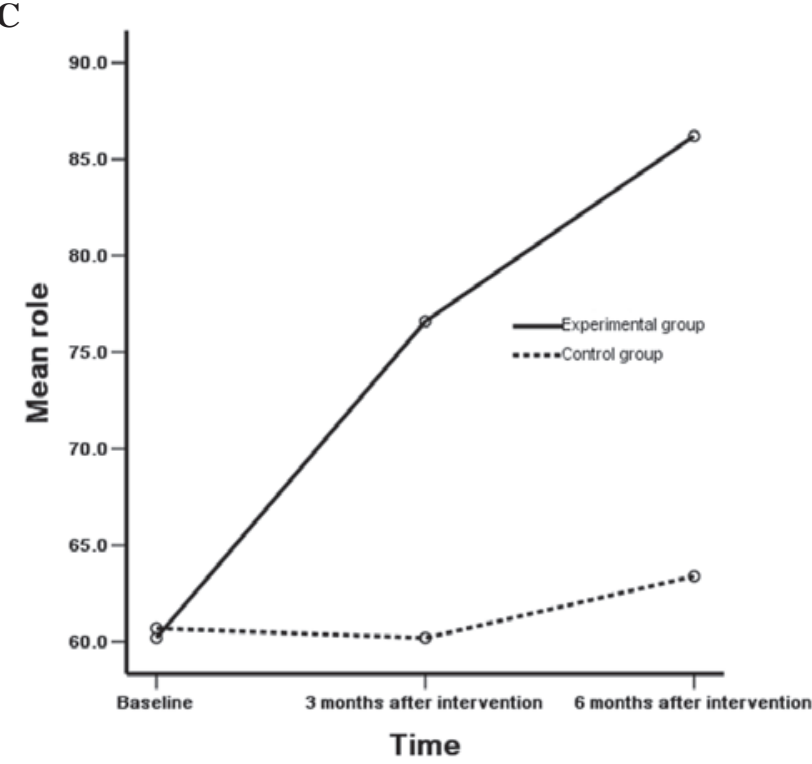

E

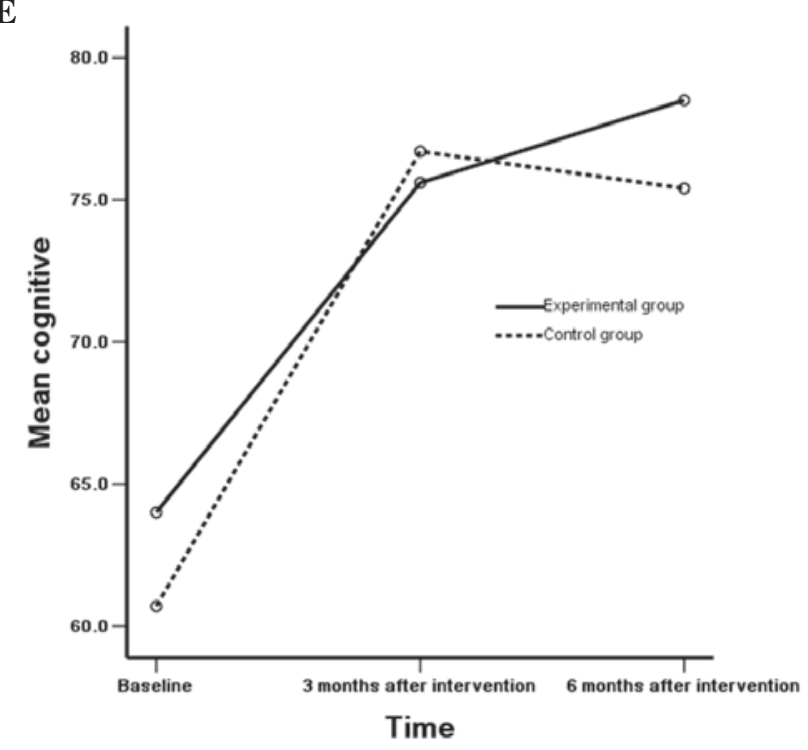

B

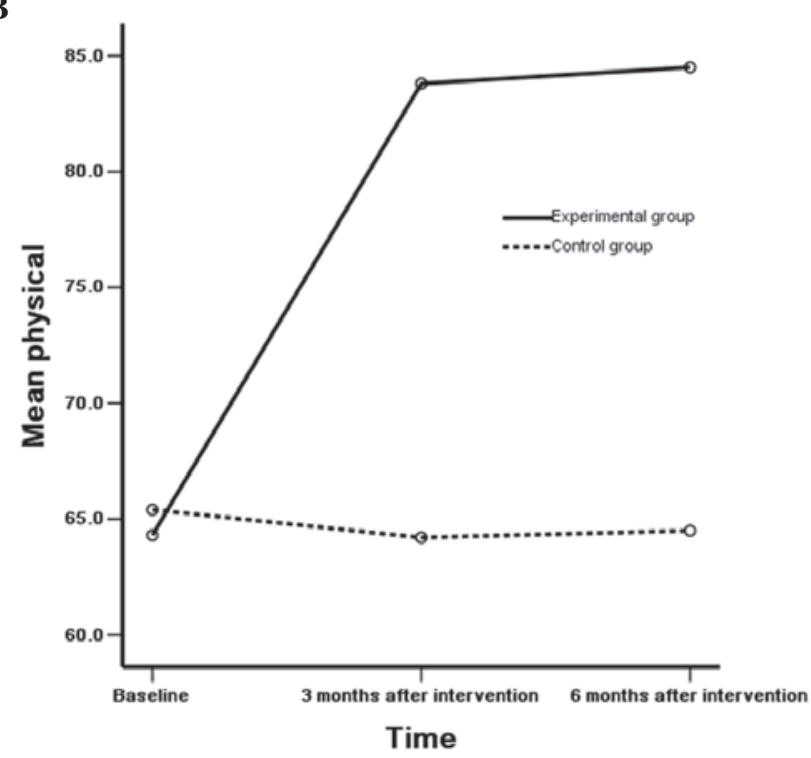

D

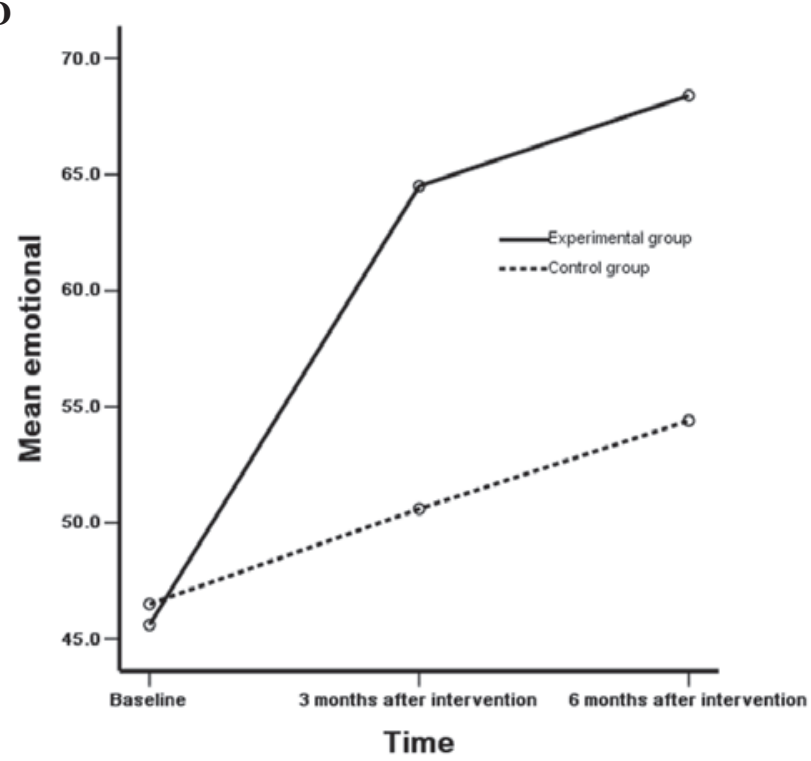

F

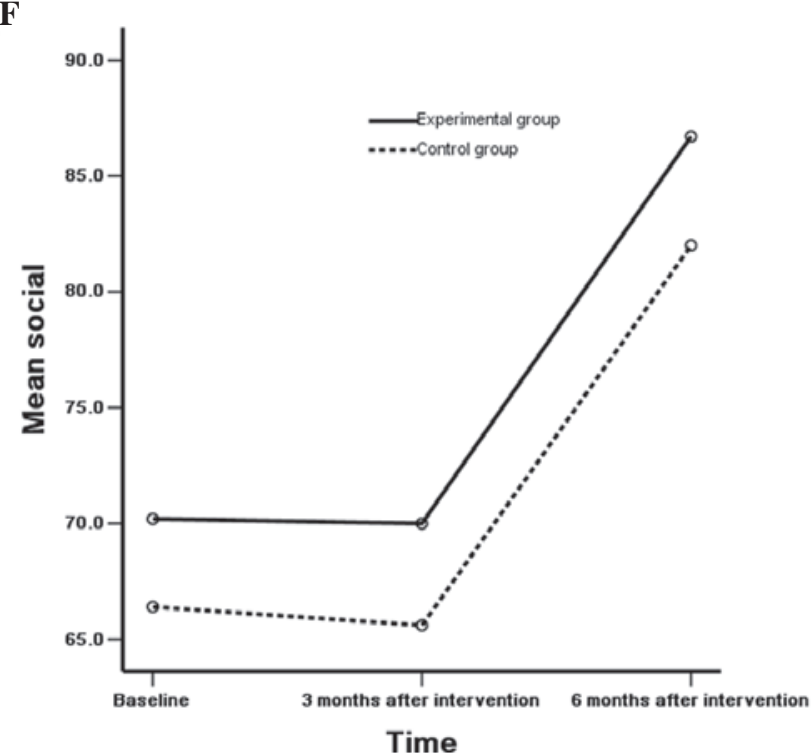

Figure 1. Outcomes of (A) global, (B) physical, (C) role, (D) emotional, (E) cognitive and (F) social difficulties of EORTC QLQ-C30 scales at different time points in the two groups are shown. 
G

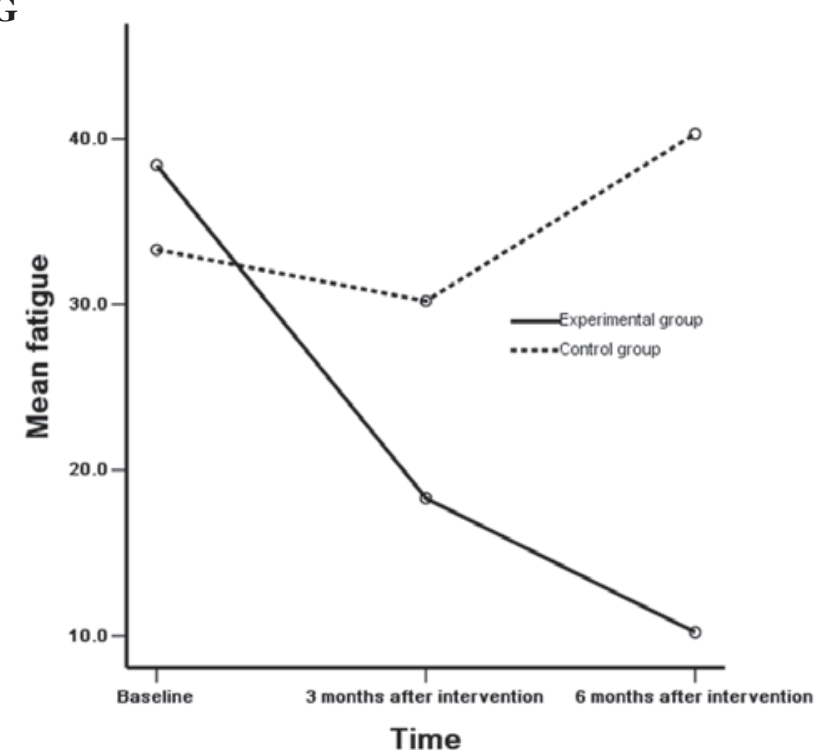

I

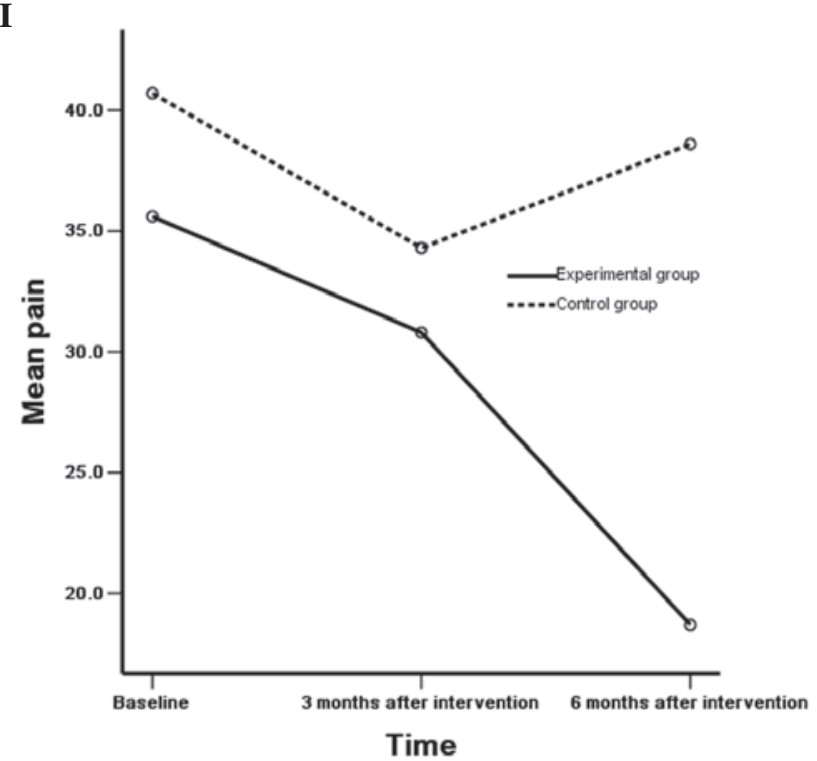

$\mathbf{K}$

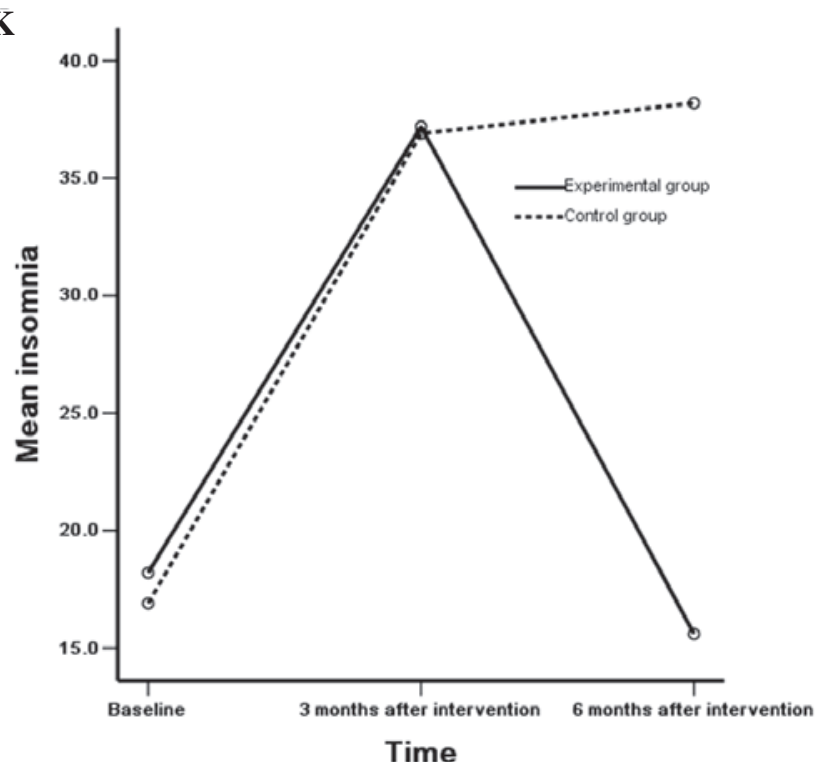

H

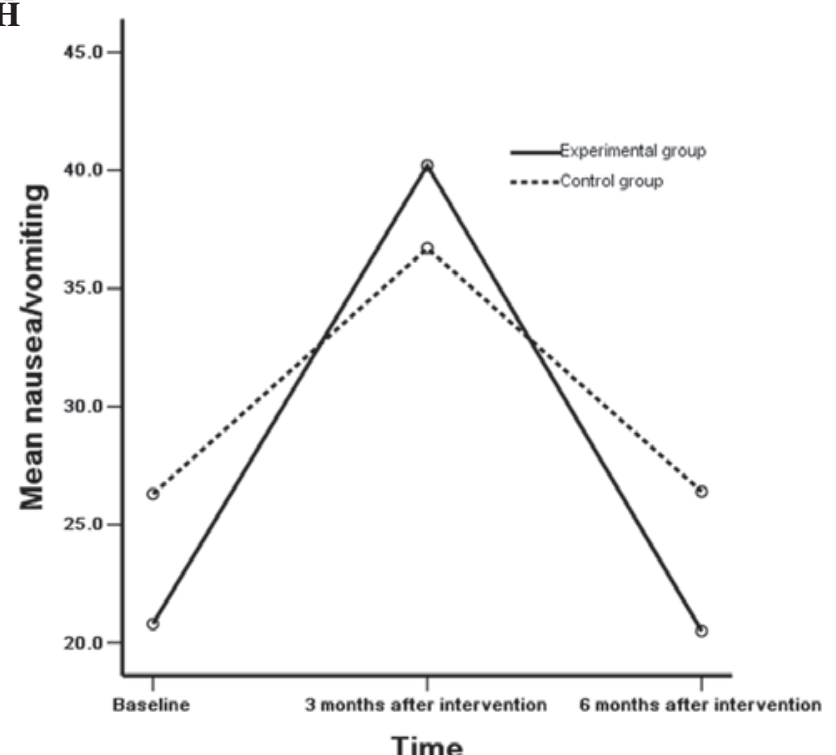

$\mathbf{J}$

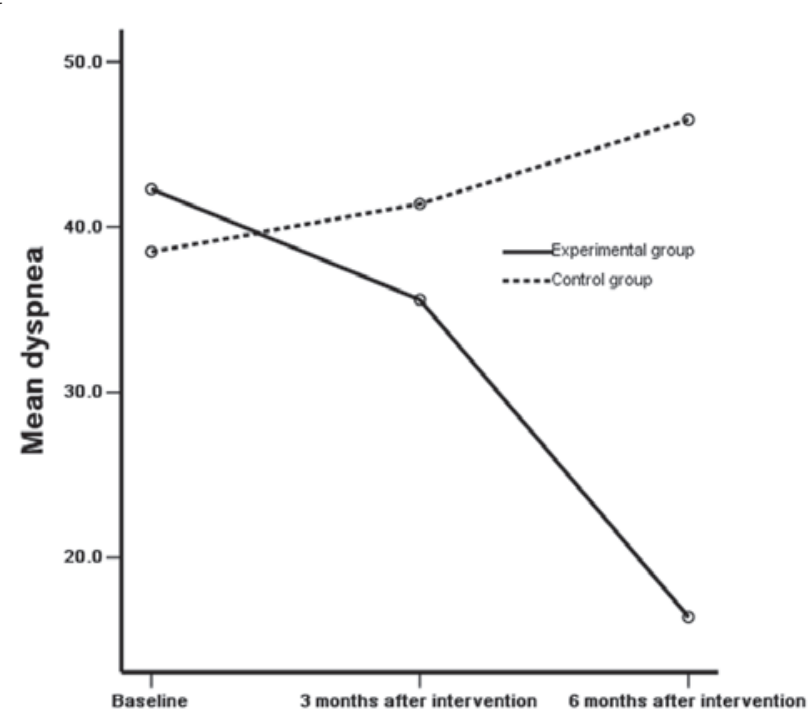

Time

$\mathbf{L}$

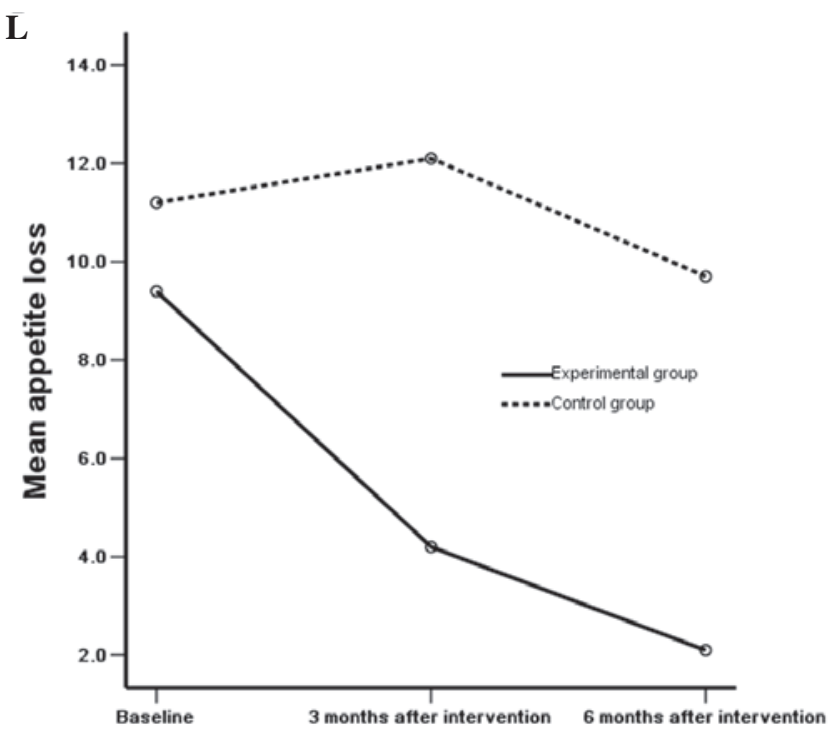

Time

Figure 1. Continued. Outcomes of (G) fatigue, (H) nausea/vomoting, (I) pain, (J) dyspnea, (K) insomnia and (L) appetite loss difficulties of EORTC QLQ-C30 scales at different time points in the two groups are shown. 

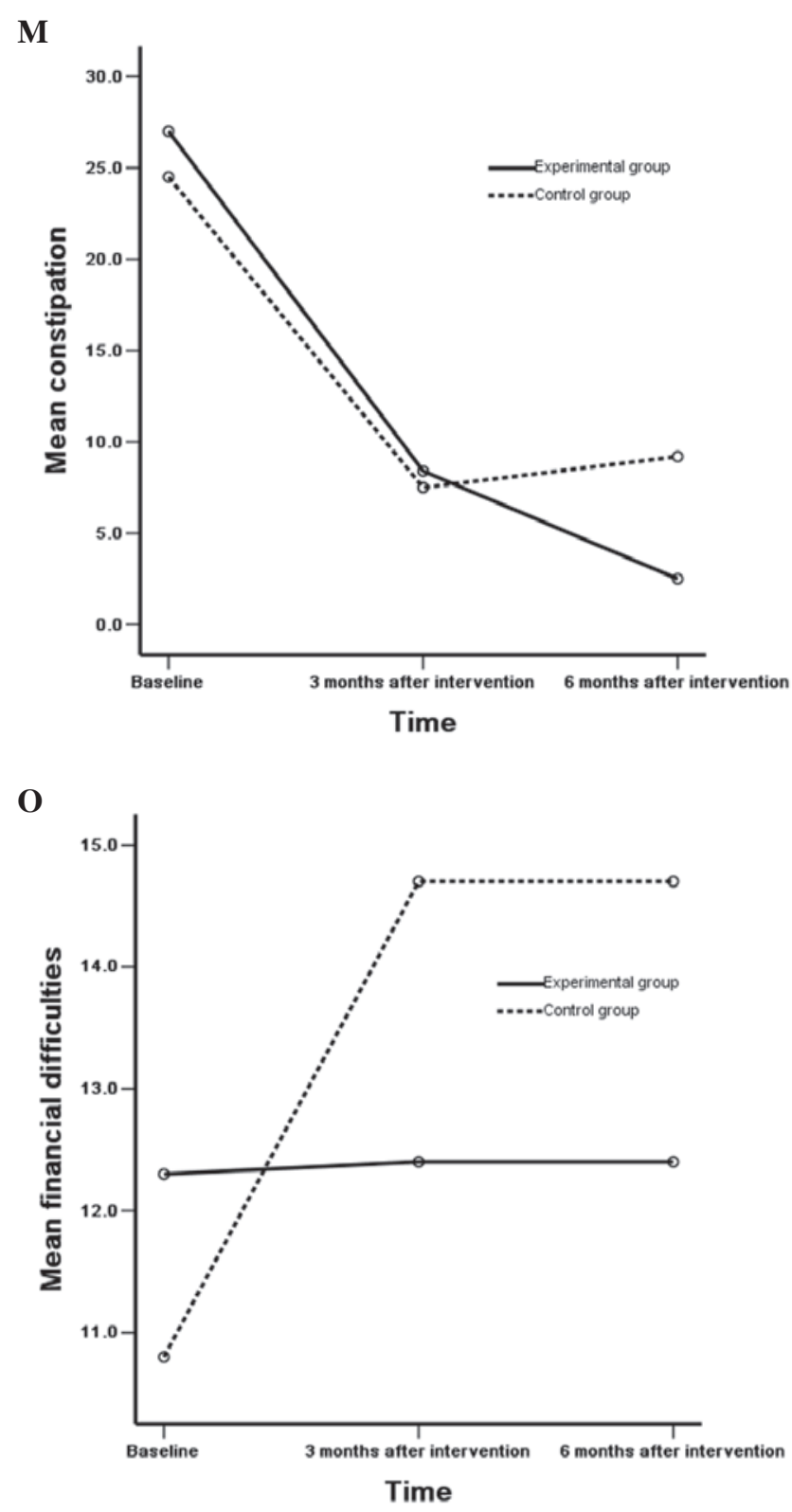

complications and health status deterioration, while the patients who underwent the systematic rehabilitation program had beneficial effects on a wide variety of physical fitness and QOL end-points including functional capacity, muscular strength, flexibility, fatigue, nausea, diarrhea, pain, physical and functional well-being, as well as overall QOL. This has been crucial in overcoming pain and distress, health status and health-related QOL.

Physical exercise has been of vital importance in the rehabilitation of oncology patients. However, the issue of how to provide guidelines for exercise prescription to the specific needs of cancer patients at various stages of the disease process has yet to be fully elucidated. Regardless of an individual being newly diagnosed, currently under treatment or in a long- or short-term survival phase, rehabilitation activity and exercise are effective adjuvant interventions for them. However, exercise prescription must be highly individualized due to the extreme variability of the cancer effects and treatment regimens on functional capacity. Exercise dosage was based on the Surgeon

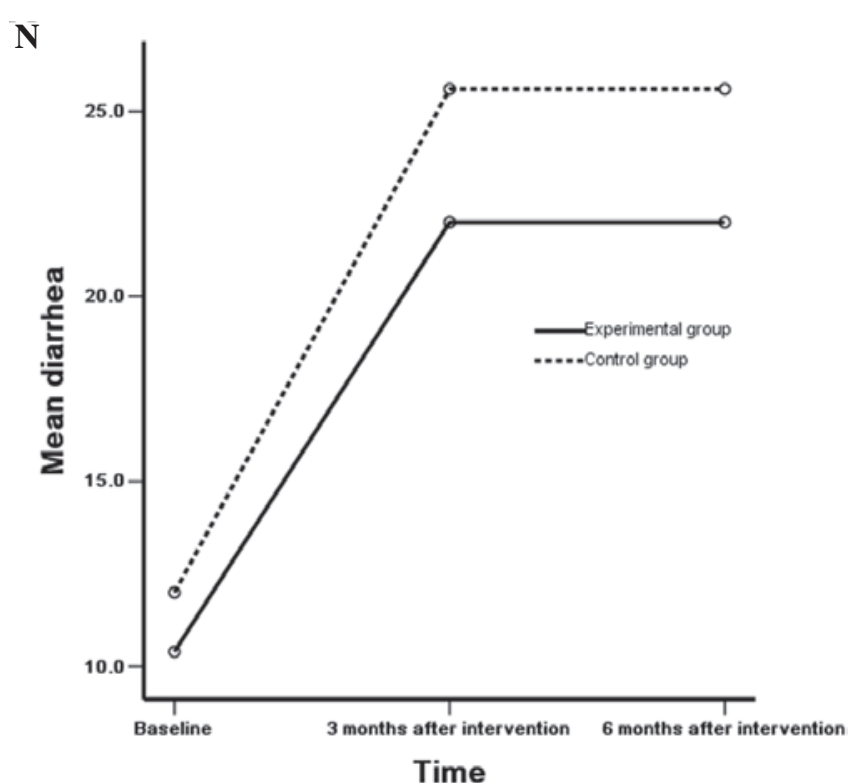

Figure 1. Continued. Outcomes of $(\mathrm{M})$ constipation, $(\mathrm{N})$ diarrhea and (O) finacial difficulties of EORTC QLQ-C30 scales at different time points in the two groups are shown.
General's 1996 recommendations (23) and the American College of Sports Medicine (24). Concerning exercise mode, walking and cycling are recommended as safe and generally well-tolerated exercise modes involving large muscle groups, with a recommended frequency of 3-5 times/week. Patients with an elevated degree of decondition should begin with daily sessions of shorter duration and lower intensity. In general, moderate intensity exercise (50-75\% heart rate reserve, Ratings of Perceived Exertion 11-14) (25) sessions of 20- to 30-min duration are recommended, with modifications as required, including extremely short exercise bouts (3-5 $\mathrm{min}$ ) followed by rest periods. The key to determine an optimal exercise dosage was not only one, such parameters would presumably be demanding for late-stage (i.e., II and III) cancer patients, as well as for those experiencing especially debilitating treatment side-effects of chemotherapy, radiation and other aspects. This should be determined, not only by the subsequent course of the disease, but by patient motivation and abilities as well. For example, a patient with stage II lung cancer might be unable 
to contemplate walking due to muscle disuse atrophy, surgical lung excision and dyspnea 1 week after surgery. Treatment could focus simply on breathing and associated dyspnea with vicinity of 1 MET. Patients are able to tolerate slow walking without frustration, anxiety or fatigue 2 weeks after surgery. After 4 weeks, patients were focused on better rehabilitation goals and were able to initiate or sustain moderate levels of activity, such as jogging and swimming.

QOL is an important factor with physical well-being and is as meaningful to patients as the actual length of life. Two validated QOL instruments were EORTC QLQ-C30 and Short Form-36 Health Survey (SF-36). EORTC provided a more detailed evaluation of lung-specific symptoms. For this reason, EORTC should be regarded as the instrument of choice for measuring QOL in patients with pulmonary resection for NSCLC (3).

Generally, the rehabilitation effect appeared to be a positive effect on the functional scales of general QOL in the experimental group 3 and 6 months after the intervention. The effects on the QOL were correlated with the desired response and were detectable after longer time periods.

In conclusion, systematic rehabilitation programs have been involved in the comprehensive management of patients undergoing lung resection of malignant lung lesions and improved pulmonary vessels as well as QOL. Preliminary evidence in this area supports the theory that systematic rehabilitation programs may be considered to be incorporated into the multidisciplinary management of patients diagnosed with lung cancer especially regarding their long-term survival.

\section{Acknowledgements}

This study was supported by the Natural Science Foundation of Hunan, China (grant no. 08jj6010).

\section{References}

1. Becerra M, John G, Drepper M, et al: Hospital-based internal medicine: a review of 2011. Rev Med Suisse 8: 259-263, 2012 (In French).

2. Carr LL, Finigan JH and Kern JA: Evaluation and treatment of patients with non-small cell lung cancer. Med Clin North Am 95: 1041-1054, 2011

3. Cocks K, King MT, Velikova G, Martyn St-James M, Fayers PM and Brown JM: Evidence-based guidelines for determination of sample size and interpretation of the European Organisation for the Research and Treatment of Cancer Quality of Life Questionnaire Core 30. J Clin Oncol 29: 89-96, 2011.

4. Soo RA, Kawaguchi T, Loh M, et al: Differences in outcome and toxicity between Asian and caucasian patients with lung cancer treated with systemic therapy. Future Oncol 8: 451-462, 2012.

5. Sloan JA, Zhao X, Novotny PJ, et al: Relationship between deficits in overall quality of life and non-small-cell lung cancer survival. J Clin Oncol 30: 1498-1504, 2012.

6. Qiao Y, Qiu X and Zhou Q: Pulmonary rehabilitation in the management of patients with lung cancer. Zhongguo Fei Ai Za Zhi 14: 744-748, 2011 (In Chinese).
7. Glattki GP, Manika K, Sichletidis L, Alexe G, Brenke R and Spyratos D: Pulmonary rehabilitation in non-small cell lung cancer patients after completion of treatment. Am J Clin Oncol 35: 120-125, 2012.

8. Jones LW: Physical activity and lung cancer survivorship. Recent Results Cancer Res 186: 255-274, 2011.

9. Andersen AH, Vinther A, Poulsen LL and Mellemgaard A: Do patients with lung cancer benefit from physical exercise? Acta Oncol 50: 307-313, 2011.

10. Jones LW, Eves ND, Peterson BL, et al: Safety and feasibility of aerobic training on cardiopulmonary function and quality of life in postsurgical non-small cell lung cancer patients: a pilot study. Cancer 113: 3430-3439, 2008.

11. Nagamatsu Y, Iwasaki Y, Hayashida R, et al: Factors related to an early restoration of exercise capacity after major lung resection. Surg Today 41: 1228-1233, 2011.

12. Benzo R, Wigle D, Novotny P, et al: Preoperative pulmonary rehabilitation before lung cancer resection: results from two randomized studies. Lung Cancer 74: 441-445, 2011.

13. Op den Kamp CM, Langen RC, Minnaard R, et al: Pre-cachexia in patients with stages I-III non-small cell lung cancer: systemic inflammation and functional impairment without activation of skeletal muscle ubiquitin proteasome system. Lung Cancer 76: 112-117, 2012.

14. Peddle-McIntyre CJ, Bell G, Fenton D, McCargar L and Courneya KS: Feasibility and preliminary efficacy of progressive resistance exercise training in lung cancer survivors. Lung Cancer 75: 126-132, 2012.

15. Quist M, Rørth M, Langer S, et al: Safety and feasibility of a combined exercise intervention for inoperable lung cancer patients undergoing chemotherapy: a pilot study. Lung Cancer 75: 203-208, 2012

16. Litterini AJ and Jette DU: Exercise for managing cancer-related fatigue. Phys Ther 91: 301-304, 2011.

17. Rueda JR, Solà I, Pascual A and Subirana Casacuberta M: Non-invasive interventions for improving well-being and quality of life in patients with lung cancer. Cochrane Database Syst Rev 7: CD004282, 2011.

18. Granger CL, McDonald CF, Berney S, Chao C and Denehy L: Exercise intervention to improve exercise capacity and health related quality of life for patients with non-small cell lung cancer: a systematic review. Lung Cancer 72: 139-153, 2011.

19. Arbane G, Tropman D, Jackson D and Garrod R: Evaluation of an early exercise intervention after thoracotomy for non-small cell lung cancer (NSCLC), effects on quality of life, muscle strength and exercise tolerance: randomised controlled trial. Lung Cancer 71: 229-234, 2011.

20. Jones LW, Hornsby WE, Goetzinger A, et al: Prognostic significance of functional capacity and exercise behavior in patients with metastatic non-small cell lung cancer. Lung Cancer 76: 248-252, 2012.

21. Na HK and Oliynyk S: Effects of physical activity on cancer prevention. Ann N Y Acad Sci 1229: 176-183, 2011.

22. Emaus A and Thune I: Physical activity and lung cancer prevention. Recent Results Cancer Res 186: 101-133, 2011.

23. Erlichman J, Kerbey AL and James WP: Physical activity and its impact on health outcomes. Paper 1: the impact of physical activity on cardiovascular disease and all-cause mortality: an historical perspective. Obes Rev 3: 257-271, 2002.

24. Schmitz KH, Courneya KS, Matthews C, et al; American College of Sports Medicine: American College of Sports Medicine roundtable on exercise guidelines for cancer survivors. Med Sci Sports Exerc 42: 1409-1426, 2010.

25. Russell WD: On the current status of rated perceived exertion. Percept Mot Skills 84: 799-808, 1997. 